\title{
CARACTERIZACIÓN DEL PERRO DE AGUA DEL CANTÁBRICO
}

\author{
CHARACTERIZATION OF THE CANTABRIC WATERDOG
}

\author{
Méndez, S. ${ }^{1}$, Dunner, S. ${ }^{1}$, García, J.A. ${ }^{2}$, de Argüello, S. ${ }^{2}$, Crespo, M.J. ${ }^{2}$, Chomón, N. ${ }^{2}$, \\ Calderón, L.A. ${ }^{2}$, Sañudo, B. ${ }^{2}$ y Cañón, J. ${ }^{1 *}$
}

1'Departamento de Producción Animal. Facultad de Veterinaria. 28040 Madrid. España. *jcanon@vet.ucm.es ${ }^{2}$ Consejería de Desarrollo Rural, Ganadería, Pesca y Biodiversidad. Santander. España.

\section{Palabras clave adicionales}

Diversidad genética. Microsatélites.

\section{RESUMEN}

El Perro de Agua del Cantábrico es una población ancestral, cuyos orígenes pueden ser comunes a los del Barbet, que se usaba en labores pesqueras. Morfológicamente se diferencia del estándar del Perro de Agua Español. Se usó la información proporcionada por 21 marcadores microsatélites en 21 muestras al azar de la población de Perro de Agua del Cantábrico, junto a 108 muestras adicionales de razas próximas (Perro de Agua Español, Portugués, Romagnol, Barbet y Caniche). Se calcularon los parámetros de diversidad genética, los estadísticos de Wright, y se analizó la existencia de una estructura genética subyaciendo a la información molecular utilizada. Genéticamente, resultó evidente la diferenciación con el resto de razas de agua próximas $\left(\mathrm{F}_{\mathrm{ST}}\right.$ medio $0,13)$. El uso de reproductores dentro de la población ha dado lugar a una gran similitud genética, sin que se aprecie la existencia de subpoblaciones.

\section{SUMMARY}

The canine breed Perro de Agua del Cantábrico is an ancestral population which probably share a common origin with the Barbet breed, and was used in fishing activities. Morphological traits in this breed are different from those in the Perro de Agua Español. We used the information provided by 21 microsatellite markers on 21 random individuals belonging to the Perro de Agua del Cantábrico and on 108 individuals belonging to other close related breeds (Perro de Agua Español, Portugues, Romagnol, Barbet and Caniche). Genetic diversity parameters and Wrigth statistics were calculated,

Presentado al Congreso SERGA (2010, Asturias).
AdDitionAl KEY WORDS

Genetic diversity. Microsatellite marker.

and population structure on the basis of multilocus genotypes was investigated. A clear genetic differentiation of the Perro de Agua del Cantábrico breed from the rest of the breeds was detected (average $\mathrm{F}_{\mathrm{ST}}$ was 0.13 ). The reproductive isolation of this population has had other genetic consequences as the great genetic homogeneity without the existence of a sub-population structure.

\section{INTRODUCCIÓN}

El Perro de Agua del Cantábrico (antes perro de lanas), como otras razas de la Península, constituye una población ancestral, cuyos orígenes pueden ser comunes a los del Barbet (www.rsce.es), y están asignados dentro de la Fédération Cynologique Internationale (www.fci.be) al Grupo VIII. En la mayoría de los pueblos cantábricos se usaba en labores pesqueras y ha formado parte de su cultura, tradiciones y modo de vivir. Desde mediados del siglo XX declina progresivamente, siendo hoy escasos los perros embarcados, aunque son numerosos en los puertos como perro de compañía.

En general, se trata de animales más ligeros y de menor estatura que los de la raza española. Así, el $75 \%$ de los machos y el $38 \%$ de las hembras quedarían excluidos del estándar racial por la alzada a la cruz, mientras que si se utiliza el criterio del peso, quedarían excluidos del estándar racial el $91 \%$ de los machos y el $80 \%$ de las hembras. 
El censo de animales de esta población en el RACIC de la Consejería de Desarrollo Rural, Ganadería, Pesca y Biodiversidad en febrero de 2009 era de 1216. El objetivo de este trabajo es analizar su posición relativa y grado de aislamiento genético respecto a las más próximas razas de perros de agua para buscar una posible justificación de su reconocimiento oficial como raza canina.

\section{MATERIAL Y MÉTODOS}

Se ha utilizado un conjunto de 21 marcadores recomendados por el ISAG (International Society of Animal Genetics), cuyas características técnicas se pueden encontrar en: http://www.isag.org.uk/ISAG/all/ 2005ISAGPanelDOG.pdf.

Además de las 21 muestras de la población objeto de estudio, se han usado los análisis de otras dos razas de la Península Ibérica: Perro de Agua Español y Portugués, y otras tres razas de perros de agua, Caniche, Barbet y Romagnol, proporcionados por G. Leroy a partir de su tesis doctoral Diversité génétique et gestion génétique des races canines (tabla I).

El cálculo de los parámetros de diversidad genética y de los estadísticos $F$ de Wright $\left(F_{I T}, F_{S T} y F_{I S}\right)$ se llevó a cabo mediante los programas Genetix v. 4.05 (Belkhir et al., 2001). Las relaciones genéticas entre

Tabla I. Características de las poblaciones. (Characteristics of populations).

\begin{tabular}{lccccc}
\hline Raza de perro & $\mathrm{NI}$ & $\mathrm{He}$ & $\mathrm{Ho}$ & $\mathrm{NA}$ & $\mathrm{F}_{\text {IS }}$ \\
\hline Agua del Cantábrico & 21 & 0,68 & 0,71 & 5,7 & $-0,02^{\mathrm{NS}}$ \\
Agua Español & 27 & 0,72 & 0,65 & 6,0 & 0,07 \\
Agua Portugués & 14 & 0,65 & 0,60 & 4,8 & 0,09 \\
Barbet & 20 & 0,70 & 0,72 & 5,4 & $-0,03^{\mathrm{NS}}$ \\
Caniche & 24 & 0,72 & 0,60 & 6,0 & 0,18 \\
Agua Romagnol & 23 & 0,65 & 0,67 & 5,2 & $-0,02^{\mathrm{NS}}$ \\
\hline
\end{tabular}

NI: Número individuos; He: Heterocigosis esperada; Ho: Heterocigosis observada; NA: Número de alelos. ${ }^{N S}$ valores no diferentes de 0 para $p=0,01$.

Archivos de zootecnia vol. 60, núm. 231, p. 406.
Tabla II. Distancia genética $\left(F_{S T}\right)$ entre cada raza con las demás y con el conjunto (Media). (Pair-wise $\mathrm{F}_{\mathrm{ST}}$ genetic distances. Media: Average genetic distance from each breed to the rest).

\begin{tabular}{lcccccc}
\hline & PAE & PAP & B & C & R & Media \\
\hline PAC & 0,12 & 0,13 & 0,12 & 0,12 & 0,16 & 0,13 \\
PAE & & 0,14 & 0,14 & 0,13 & 0,14 & 0,13 \\
PAP & & & 0,11 & 0,11 & 0,18 & 0,13 \\
B & & & & 0,09 & 0,15 & 0,12 \\
C & & & & & 0,15 & 0,12 \\
PAR & & & & & & 0,16
\end{tabular}

PAC: Perro de Agua del Cantábrico; PAE: Perro de Agua Español; PAP: Perro de Agua Portugués; B: Barbet; C: Caniche; PAR: Perro de Agua Romagnol. Todos los valores diferentes de 0 para $p=0,01$.

las razas se representaron gráficamente mediante un algoritmo neighbor-joining a partir de la distancia genética $F_{S T}$. El programa Genetix también se utilizó para realizar el análisis multivariante de correspondencia. Se utilizó el método de inferencia bayesiana implementado en el programa Structure (Pritchard et al., 2000) para ver en que medida los individuos de la población estudiada se asignaban o no a la propia población.

\section{RESULTADOSYDISCUCIÓN}

Las estimaciones de los principales parámetros de diversidad se presentan en la tabla I. Hay tres poblaciones de perros de agua que aparentemente están en equilibrio genético (Hardy-Weinberg): Cantábrico, Romagnol y Barbet. Las otras tres presentaron niveles significativos de consanguinidad, posiblemente como consecuencia de subdivisión genética dentro de esas razas.

En cuanto a los valores globales de sub-división, las estimaciones de los estadísticos de Wrigth proporcionaron unos valores para $\mathrm{F}_{\mathrm{IT}}, \mathrm{F}_{\mathrm{ST}}$ y $\mathrm{F}_{\mathrm{IS}}$ de $0,17(0,15$ $0,20), 0,13(0,11-0,15)$, y $0,05(0,03-0,07)$ 
Tabla III. Proporción de genoma de cada población analizada que proviene de cada uno de los hipotéticos grupos genéticos. (Average proportions of breed genomes belonging to the inferred genetic clusters).

\begin{tabular}{|c|c|c|c|c|c|c|c|c|c|c|c|c|}
\hline & \multicolumn{2}{|c|}{ Dos grupos } & \multicolumn{4}{|c|}{ Cuatro grupos } & \multicolumn{6}{|c|}{ Seis grupos } \\
\hline & 1 & 2 & 1 & 2 & 3 & 4 & 1 & 2 & 3 & 4 & 5 & 6 \\
\hline PAC & 0,02 & 0,98 & 0,02 & 0,01 & 0,97 & 0,01 & 0,01 & 0,04 & 0,02 & 0,89 & 0,01 & 0,03 \\
\hline PAE & 0,02 & 0,98 & 0,89 & 0,01 & 0,08 & 0,03 & 0,01 & 0,06 & 0,02 & 0,02 & 0,01 & 0,88 \\
\hline PAP & 0,30 & 0,70 & 0,06 & 0,02 & 0,71 & 0,21 & 0,02 & 0,93 & 0,01 & 0,01 & 0,01 & 0,02 \\
\hline B & 0,98 & 0,02 & 0,02 & 0,02 & 0,01 & 0,95 & 0,93 & 0,02 & 0,01 & 0,01 & 0,02 & 0,01 \\
\hline $\mathrm{C}$ & 0,98 & 0,02 & 0,01 & 0,01 & 0,01 & 0,97 & 0,16 & 0,02 & 0,79 & 0,02 & 0,01 & 0,01 \\
\hline PAR & 0,98 & 0,02 & 0,01 & 0,95 & 0,02 & 0,02 & 0,02 & 0,01 & 0,01 & 0,02 & 0,95 & 0,01 \\
\hline
\end{tabular}

PAC: Perro de Agua del Cantábrico; PAE: Perro de Agua Español; PAP: Perro de Agua Portugués; B: Barbet; C: Caniche; PAR: Perro de Agua Romagnol.

respectivamente (entre paréntesis los intervalos de confianza del 95\%).

Resulta evidente la subdivisión que se aprecia, con un valor medio de $\mathrm{F}_{\mathrm{ST}}$ del $13 \%$ (las razas explican el 13 por 100 de la diversidad genética global medida).

Es interesante analizar en más detalle este valor global de diferenciación genética entre las razas incluidas. Para ello se puede recurrir a estimar las distancias genéticas por parejas de razas (tabla II).

El Romagnol aparece el más aislado de todos, y las razas Barbet y Caniche las más próximas.El Perro de Agua del Cantábrico

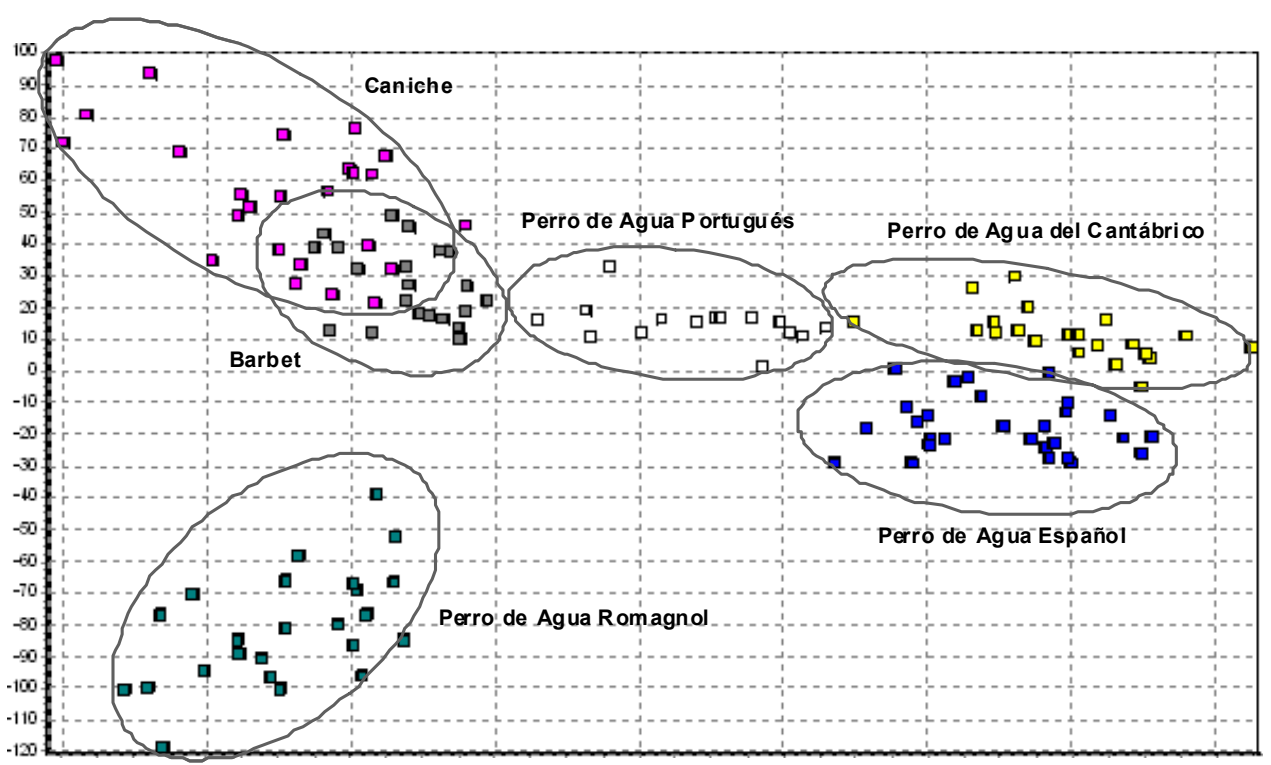

Figura 1. Posición relativa, que corresponde a los dos ejes de mayor inercia, obtenidos utilizando los marcadores moleculares como variables explicativas mediante un análisis de correspondencia. (Relative position of the populations using the two axes with higher inertia obtained through a correspondence analysis and the alleles as independent variables). 


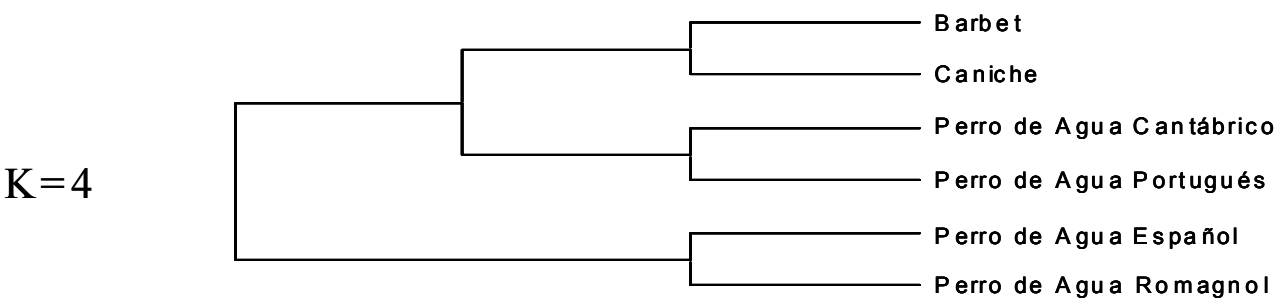

Figura 2. Dendrogramas (neighbour-joining) de matrices de distancias entre parejas de poblaciones basadas en el parecido de los porcentajes de genoma que comparten entre el número de poblaciones ancestrales $(K)$ decidido a priori. (Neighbour-joining tree of genetic distances based on estimated membership coefficients $\mathrm{q}_{k}(\mathrm{i})$ of breed $\mathrm{i}$ for cluster $\mathrm{k}$ ).

aparece tan alejado (próximo) del Perro de Agua Español como del Barbet y Caniche.

En la figura 1, se presenta el resultado obtenido mediante análisis multivariante utilizando las dos componentes más explicativas. Se puede apreciar la posición relativa que ocupan los ejemplares de cada una de las razas. Sólo hay importante solapamiento entre las razas Caniche y Barbet.

Finalmente, se utilizó un algoritmo de agrupación basado en un modelo resuelto mediante inferencia bayesiana. El resumen puede analizarse en la tabla III o en la figura 2 en la que aparecen las proporciones de genoma que para cada población (individuo) muestreada proviene de cada una de las cuatro hipotéticas poblaciones ancestrales consideradas.

Aunque se puede observar una gran homogeneidad genética, la mayor parte del genoma de cada población se asigna a un único grupo u origen, en algunos casos, como en el Perro de Agua Portugués cuando el número de grupos es bajo, 2 o 4, o en el Caniche cuando el número de grupos es elevado, el genoma de la raza se distribuye entre dos orígenes. El Perro de Agua del

\section{BIBLIOGRAFÍA}

Belkhir, K., Borsa, P., Chikhi, L., Raufaste, N. and Bonhomme, F. 2001. Genetix, logiciel sous Windows TM pour la génétique des populations, laboratoire génome, populations, interactions. CNRS UPR 9060. Université de Montpellier II.
Cantábrico manifiesta una gran homogeneidad genética, el $90 \%$ o más de su genoma se asigna a un único origen que, para el caso de valor K superior a 5 es distinto a los orígenes Portugués o Español.

Se puede mostrar el parecido entre las poblaciones analizadas con el criterio del porcentaje de genoma común entre las poblaciones definidas a priori (figura 2).

En conclusión, la población canina Perro de Agua del Cantábrico muestra evidencias que confirman su arraigo social, cultural, histórico y geográfico, y una clara diferenciación morfológica y genética que permite discriminaarla de otras poblaciones caninas del mismo grupo con distribución por áreas geográficas próximas.

\section{AGRADECIMIENTOS}

Asociación Amigos del Perro de Agua del Cantábrico por gran parte del material utilizado en este trabajo, y a Grégoire Leroy del Dpto. de Génétique et Diversité Animales en la Universidad de AgroParis Tech. por la información genética de las razas Barbet, Caniche y Romagnol.

Montpellier. France. http://www.univ-montp2.fr/ $\sim$ genetix/genetix/genetix.htm

Pritchard, J.K., Stephens, M. and Donnelly, P. 2000. Inference of population structure using multilocus genotype data. Genetics, 155: 945-959. 